\title{
Effects of Integrated Health Management Intervention on Overweight and Obesity
}

\author{
Yiting Yang, ${ }^{1}$ Chung Wah Ma, ${ }^{1}$ Yide Yang, ${ }^{2}$ Xiaoling Wang, ${ }^{1}$ Xiaoliang Lin,, \\ Lianguo Fu, ${ }^{3}$ Shuo Wang, ${ }^{2}$ Zhongping Yang, ${ }^{2}$ Zhenghe Wang, ${ }^{2}$ Xiangkun Meng, ${ }^{2}$ \\ Dongmei $\mathrm{Ma}^{2}$ Rui $\mathrm{Ma},{ }^{2}$ and Jun $\mathrm{Ma}^{2}$ \\ ${ }^{1}$ Research \& Development Centre, Infinitus (China) Company Ltd., Guangzhou, China \\ ${ }^{2}$ Institute of Child and Adolescent Health, School of Public Health, Peking University Health Science Center, Beijing, China \\ ${ }^{3}$ Department of Preventive Medicine, Bengbu Medical College, Bengbu City, Anhui, China
}

Correspondence should be addressed to Jun Ma; majunt@bjmu.edu.cn

Received 20 June 2016; Revised 6 November 2016; Accepted 14 November 2016; Published 1 January 2017

Academic Editor: Omar Said

Copyright (C) 2017 Yiting Yang et al. This is an open access article distributed under the Creative Commons Attribution License, which permits unrestricted use, distribution, and reproduction in any medium, provided the original work is properly cited.

Overweight or obese adults aged 20 55 years and living in Beijing more than one year were randomly divided into different management groups. A one-year integrated health management intervention was applied in the health management groups. The physical indicators and metabolic indicators changed after one-year intervention on the overweight and obese adults. The annual reduction of the physical indicators was significant in all groups $(p<0.05)$ except the weight loss in the placebo + general management group. The health management and the dietary supplement have statistically significant $(p<0.001, p<0.001)$ effects on the annual reduction of these indicators and interactive effect between them was found on some of these indicators such as bodyweight, body mass index (BMI), body fat ratio (BFR), and hipline $(p<0.05)$. The dietary supplement + health management group had the best annual reduction effects for the indicators among the groups. Integrated health management interventions including both dietary supplements intervention and health management could improve metabolic indicators in overweight and obese adults together with the physical indicators, suggesting the intermediated role of metabolic indictors in controlling obesity.

\section{Introduction}

Overweight/obesity is epidemic worldwide and also in China with the number of obese patients increasing at a significant rate each year $[1,2]$. The escalating rates of obesity parallel the increases in the number of chronic diseases such as cardiovascular disease, diabetes, and hypertension [3]. With the advances in the research of obesity and the related chronic diseases over the years, some metabolic indictors were found to correlate with these diseases [4]. For example, there is evidence that high serum C-reactive protein (CRP) is related to overweight or obese status and also involved in the development of several chronic diseases such as obesity, cardiovascular diseases, type 2 diabetes mellitus, and colorectal and other cancers [5-7]. The role of diet or exercises in reducing serum CRP and thereby modulating obesity was also supported by large body of evidences [8-10].
Therefore, changes in these metabolic indicators might affect the outcomes of overweight/obesity intervention. To date, no study has investigated the effect of integrated health management interventions including both dietary supplements intervention and health management on these metabolic indicators. Therefore, in this study an integrated health management intervention was applied and the effectiveness of intervention was evaluated by physical examinations and by measurement of different metabolic indicators.

\section{Subjects and Methods}

2.1. Subjects. Subjects were recruited voluntarily through a recruitment notice and $\mathrm{BMI}$ is used as the screening indicator. Subjects who met the following criteria were recruited: (1) males and females aged 20-55 years; (2) having lived in their 


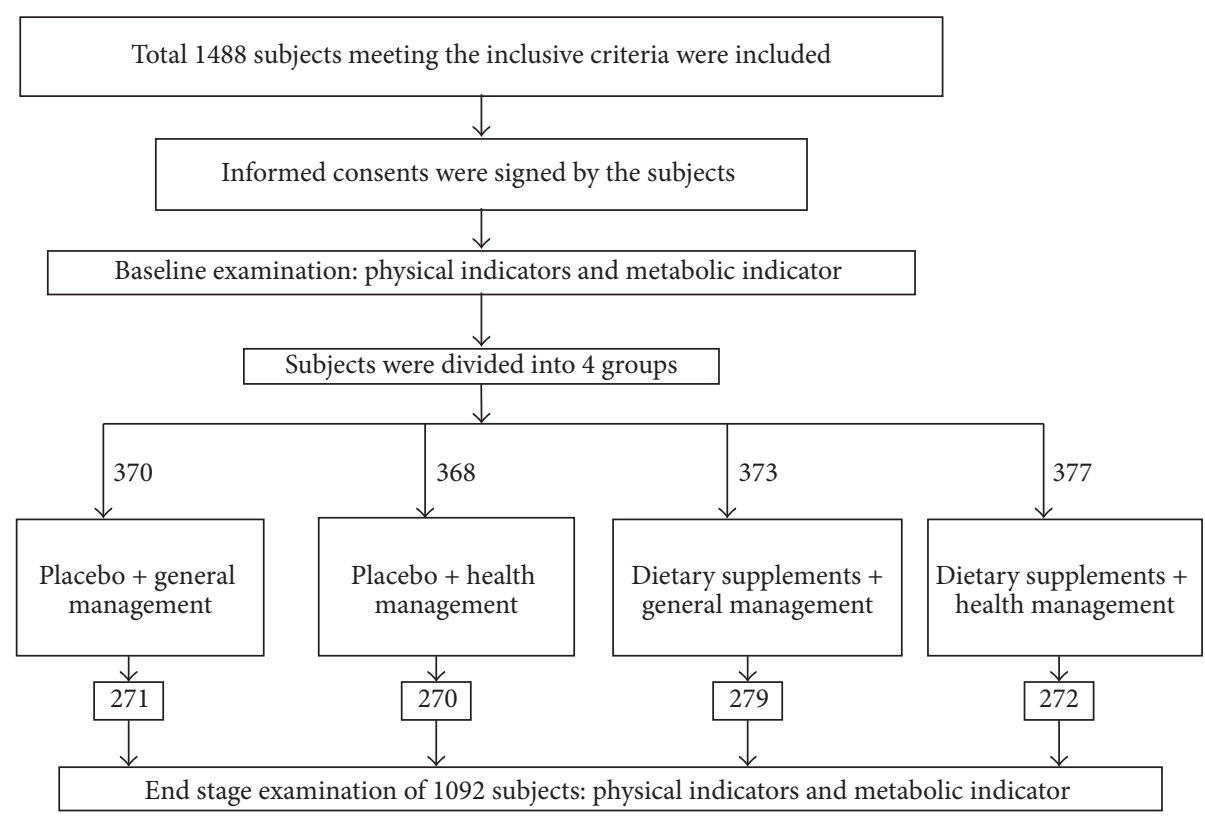

FIGURE 1: Schematic picture of the procedure of the one-year intervention.

cities for at least one year; (3) meeting the diagnostic criteria in the Guidelines for the Prevention and Control of Overweight and Obesity in Chinese Adults (2006): overweight: $24 \mathrm{~kg} / \mathrm{m}^{2} \leq \mathrm{BMI}<28 \mathrm{~kg} / \mathrm{m}^{2}$; obesity: BMI $\geq 28 \mathrm{~kg} / \mathrm{m}^{2}$. The exclusion criteria were as follows: (1) suffering from certain severe organic diseases; (2) secondary obesity arising from illness; (3) women with pregnancy plan, pregnant women, and lactating women; (4) taking any other constitutional conditioning or weight loss product before or during intervention.

2.2. Intervention Program. The intervention period was one year. Intervention measures include health management, general management, dietary supplements, and placebo. (1) Health management: health management includes nutrition, exercise, and behavioral correction. Methods such as health education, regular health checkup, telephone interview, and behavioral diary were used for integrated management and guidance. A novel "3-2-1-0" behavioral plan including main dietary and exercise actions was also set to improve the compliance of subjects and monitor the health management intervention. In this plan, “ 3 " represented eating vegetables of at least 3 fists' size per day; " 2 " represented eating meat of 2 palms' size per day; "1" represented walking 10,000 steps per day; and " 0 " represented not eating high-sugar snacks. (2) General management: general management was performed under the same physical monitoring as the health management groups without special management, offering multiple physical checkup reports. (3) Dietary supplements: the dietary supplements used in this study were extracted and refined from edible materials, such as poria and mushroom, and added with edible seasonings. In addition, the dietary supplements had constitutional conditioning effects according to Traditional Chinese Medicine. The slimming tablets could invigorate the spleen and induce diuresis, dietary fiber could supplement dietary fiber to increase satiation, GestAid Plus could regulate the stomach and spleen, Yi Rui Capsule could activate blood circulation to remove stasis, and Schizandra Plus could supplement Qi. They usually have no adverse reaction and had been certified by the State Food and Drug Administration for safety and efficacy through human and animal experiments. The dietary supplements were administrated twice a day, at lunch and supper. (4) The placebo was a preparation free from any pharmacological ingredient, prepared from edible materials, similar to the foods and health products in shape. The placebo was administrated twice a day at lunch and supper.

2.3. Intervention Grouping. Subjects were divided into 4 groups by simple randomization according to the type of intervention measurements. Group 1: placebo + general management $(\mathrm{P}+\mathrm{GM})$; Group 2: placebo + health management $(\mathrm{P}+\mathrm{HM})$; Group 3: dietary supplements + general management (DS + GM); Group 4: dietary supplements + health management (DS + HM). The dietary and placebo studies were double-blinded. Neither subjects nor the medical staffs were aware of the grouping situation. Study procedures and grouping were listed in Figure 1 and loss of samples at different stages was shown in Table 1.

2.4. Measuring Indicators. The physical indicators were measured through health checkup at baseline, month 1, month 3, month 6 , month 9, and month 12 of intervention, respectively. The indicators included height, bodyweight, body fat ratio (BFR), waistline, and hipline. Mean value was taken after measurement. Height was measured twice at each time point in $\mathrm{cm}$ and accurate to $0.1 \mathrm{~cm}$, and if error exceeded $0.5 \mathrm{~cm}$, height would be measured three times. Each subject stands 
TABLE 1: Sample sizes and loss to follow-up ratios at different stages.

\begin{tabular}{lcc}
\hline Intervention time & \multicolumn{2}{c}{ Total } \\
\hline Baseline & 1488 & Loss of follow-up (\%) \\
1 month & 1420 & - \\
3 months & 1380 & 4.57 \\
6 months & 1271 & 7.26 \\
9 months & 1186 & 14.58 \\
12 months & 1092 & 20.3 \\
\hline
\end{tabular}

upright on the base of the height meter with bare feet, no hat, and close-fitting underwear. Weight was measured twice in $\mathrm{kg}$ and accurate to $0.1 \mathrm{~kg}$. If error exceeds $0.1 \mathrm{~kg}$, it would be measured three times. Waistline and hipline were measured twice in $\mathrm{cm}$ and accurate to $0.1 \mathrm{~cm}$. They would be measured three times if error exceeds $1.0 \mathrm{~cm}$. BFR was checked by TANITA, MC-180 body composition analyzer, for any metallic implant, such as tooth brace, pacemaker, and joint and accurate to one decimal place. The 14 metabolic indicators measured at baseline, middle stage (month 6), and final stage (month 12) include high-density lipoprotein cholesterol (HDL), lowdensity lipoprotein cholesterol (LDL), triglyceride (TG) and total cholesterol (TC), leptin (LEP), adiponectin, fasting insulin level (INS), fasting blood sugar (GLU), free fatty acids (FFA), glycosylated hemoglobin (HbA1C), insulin resistance index (HOMAIR), insulin sensitivity index (HOMAIS), tumor necrosis factor $\alpha$ (TNF- $\alpha$ ), and C-reactive protein (CRP). Calculation method for key insulin-related derivative indicators (steady-state model evaluation): insulin resistance index $($ HOMAIR $)=$ fasting plasma glucose $(\mathrm{GLU}, \mathrm{mmol} / \mathrm{L})$ $\times$ fasting insulin (INS, $\mathrm{mIU} / \mathrm{L}$ )/22.5; insulin sensitivity index $($ HOMAIS $)=1 /[($ fasting plasma glucose $(\mathrm{GLU}, \mathrm{mmol} / \mathrm{L}) \times$ fasting insulin (INS, mIU/L)] [11].

2.5. Ethical Application and RCT Registration. This project has been approved by the Ethical Committee of Peking University Health Science Center. The progress report has been submitted and approved, and the ethical closing application is ongoing. The number of the ethical certificate is IRB00001052-13086. This project has been registered on ClinicalTrials.gov, with RCT ID of NCT02298426.

2.6. Statistical Analysis Methods. The SPSS20.0 software is used for a statistical analysis. Measured data is expressed in mean \pm standard deviation. The statistical methods include factorial design variance analysis, single-factor variance analysis, multiple logistic regression analysis, and chi-square test, where $p<0.05$ denotes a statistically significant difference. Data is analyzed using the Per-Protocol (PP) analysis method, and the analysis is for subjects complying with intervention only.

\section{Results}

3.1. Physical Indicators Changings. Total 1488 subjects were recruited to this study and 1092 completed. The other subjects were excluded because of the following reasons: (1) long bone density measuring time or perceived irradiation; (2) not attending physical checkup (busy working, trip, etc.); (3) poor compliance (doubting product safety, etc.); (4) loss of contact (not answering calls, shutdown, etc.); (5) physical conditions (pregnancy/readiness for pregnancy, etc.); (6) sickness of self/family member. All the 1,092 subjects in this study that completed one-year health interventions had no statistically significant difference in age, gender, and baseline BMI among the four intervention groups (data were not shown). After this one-year intervention on the overweight and obese adults, the annual reduction of the physical indicators (Table 2) such as bodyweight, BMI, BFR, hipline, and waistline was significant in all four groups $(p<0.05)$ except the weight loss $(-0.23 \mathrm{~kg})$ in the placebo + general management group. It was also found that both the health management and the dietary supplement had statistically significant $(p<0.001, p<0.001)$ effects on the annual reduction of these indicators. In addition, there was interactive effect between the health management and the dietary supplement in annual reduction of some of the key indicators such as bodyweight, body mass index (BMI), body fat ratio (BFR), and hipline $(p<0.05)$. Based on pairwise comparison, the differences between any two of the four groups are statistically significant for these indicators $(p<$ 0.05 ). The dietary supplement + health management group had the best annual reduction effects for the indicators among the four groups as shown in Table 2 with annual weight loss of $4.24 \mathrm{~kg}$, annual BMI loss of $1.60 \mathrm{~kg} / \mathrm{m}^{2}$, annual WC loss of $6.61 \mathrm{~cm}$, and annual hipline loss of $3.25 \mathrm{~cm}$. The effective rates of weight loss of the four groups are $3.30 \%, 31.11 \%, 34.78 \%$, and $42.86 \%$, respectively, with the dietary supplements + health management having the highest effective rate of annual weight loss of 5\%. Based on chi-square test, the differences among the four groups are statistically significant as in Table 2 $\left(\chi^{2}=40.02, p<0.001\right)$.

3.2. Metabolic Indicators Changings. Following the improvement of the physical indicators, the metabolic indicators had also changed after the one-year intervention. It was found from the pairwise comparison of metabolic indicators at baseline and month 12 among the four groups (Table 3) that there was no statistically significant difference among the four groups. However, there were declines of hypersensitive Creactive protein (CRP), total cholesterol (TC), triglyceride (TG), free fatty acids (FFA), blood sugar (GLU), insulin (INS), HOMA-Insulin Resistance (HOMAIR), leptin (LEP), HbAlC, low-density lipoprotein (LDL), and tumor necrosis factor (TNF) and increased level of high-density lipoprotein (HDL) and HOMA-Insulin Sensitivity (HOMAIS). From the analysis, the annual declines in C-reactive protein, leptin, triglyceride, blood sugar, and HOMAIR were the best in the dietary supplements + health management group while HDL was higher in the dietary supplements + health management group. In order to explore effects of the dietary supplements and health management program on the metabolic indicators in overweight/obese adults and their interaction, a linear mixed model was built at baseline, month 6 , and month 12 with/without dietary supplements intervention and 


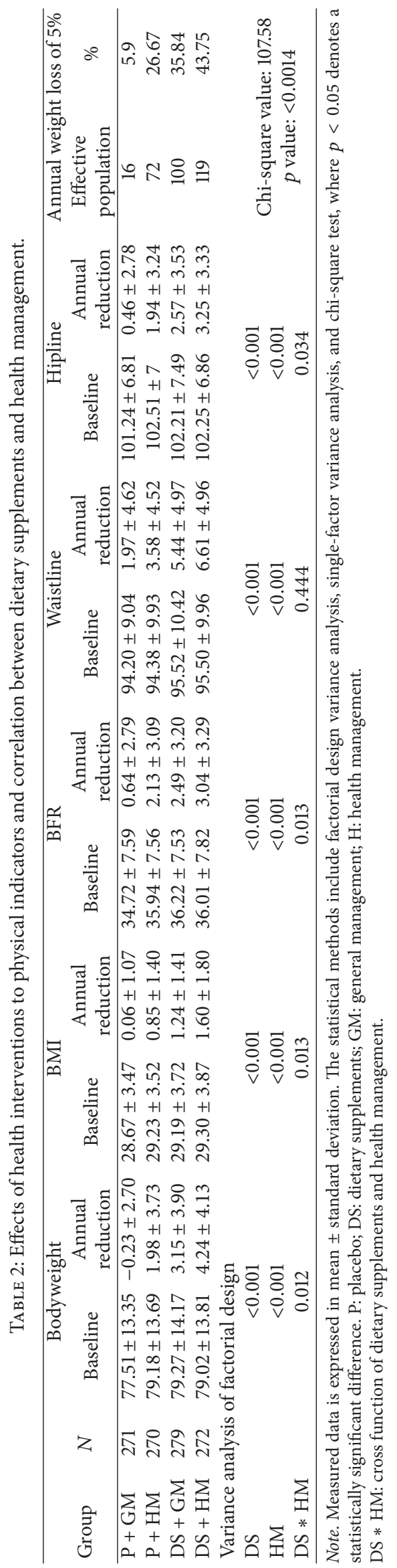


TABLE 3: Annual change of metabolic indicators after one-year intervention.

\begin{tabular}{|c|c|c|c|c|c|c|}
\hline & & $N$ & Baseline & 12 months & Annual decline & $p$ \\
\hline \multirow{4}{*}{ Lnhs-CRP } & $\mathrm{P}+\mathrm{GM}$ & 270 & $0.05 \pm 1.07$ & $0.28 \pm 1.04$ & $-0.24 \pm 0.95$ & $<0.001$ \\
\hline & $\mathrm{P}+\mathrm{HM}$ & 268 & $0.03 \pm 1.17$ & $0.24 \pm 1.17$ & $-0.15 \pm 0.96$ & 0.011 \\
\hline & $\mathrm{DS}+\mathrm{GM}$ & 277 & $0.02 \pm 1.11$ & $0.15 \pm 1.04$ & $-0.15 \pm 0.79$ & 0.002 \\
\hline & $\mathrm{DS}+\mathrm{HM}$ & 271 & $0.05 \pm 1.16$ & $0.07 \pm 1.1$ & $0 \pm 0.98$ & 0.999 \\
\hline \multirow{4}{*}{ TC } & $\mathrm{P}+\mathrm{GM}$ & 270 & $4.44 \pm 0.87$ & $4.59 \pm 0.91$ & $-0.15 \pm 0.73$ & 0.001 \\
\hline & $\mathrm{P}+\mathrm{HM}$ & 268 & $4.42 \pm 0.83$ & $4.55 \pm 0.85$ & $-0.14 \pm 0.73$ & 0.002 \\
\hline & $\mathrm{DS}+\mathrm{GM}$ & 277 & $4.52 \pm 0.88$ & $4.58 \pm 0.88$ & $-0.06 \pm 0.67$ & 0.151 \\
\hline & $\mathrm{DS}+\mathrm{HM}$ & 271 & $4.5 \pm 0.88$ & $4.67 \pm 0.91$ & $-0.17 \pm 0.67$ & $<0.001$ \\
\hline \multirow{4}{*}{ TG } & $\mathrm{P}+\mathrm{GM}$ & 268 & $1.16 \pm 1$ & $1.76 \pm 1.31$ & $-0.14 \pm 0.91$ & 0.011 \\
\hline & $\mathrm{P}+\mathrm{HM}$ & 267 & $1.64 \pm 1.2$ & $1.57 \pm 1.17$ & $0.04 \pm 0.75$ & 0.372 \\
\hline & $\mathrm{DS}+\mathrm{GM}$ & 277 & $1.57 \pm 1.04$ & $1.41 \pm 0.88$ & $0.16 \pm 0.82$ & 0.001 \\
\hline & $\mathrm{DS}+\mathrm{HM}$ & 270 & $1.55 \pm 1.03$ & $1.33 \pm 0.9$ & $0.22 \pm 0.93$ & $<0.001$ \\
\hline \multirow{4}{*}{ HDL-c } & $\mathrm{P}+\mathrm{GM}$ & 270 & $1.31 \pm 0.29$ & $1.29 \pm 0.24$ & $-0.02 \pm 0.21$ & 0.204 \\
\hline & $\mathrm{P}+\mathrm{HM}$ & 268 & $1.31 \pm 0.28$ & $1.32 \pm 0.27$ & $0.01 \pm 0.23$ & 0.677 \\
\hline & $\mathrm{DS}+\mathrm{GM}$ & 277 & $1.35 \pm 0.26$ & $1.32 \pm 0.25$ & $-0.03 \pm 0.23$ & 0.042 \\
\hline & $\mathrm{DS}+\mathrm{HM}$ & 271 & $1.32 \pm 0.26$ & $1.36 \pm 0.29$ & $0.05 \pm 0.25$ & 0.001 \\
\hline \multirow{4}{*}{ LDL-c } & $\mathrm{P}+\mathrm{GM}$ & 270 & $2.91 \pm 0.73$ & $3.07 \pm 0.85$ & $-0.03 \pm 0.68$ & 0.523 \\
\hline & $\mathrm{P}+\mathrm{HM}$ & 268 & $2.88 \pm 0.82$ & $2.98 \pm 0.88$ & $0.03 \pm 0.77$ & 0.475 \\
\hline & $\mathrm{DS}+\mathrm{GM}$ & 277 & $2.97 \pm 0.78$ & $3.15 \pm 0.89$ & $-0.01 \pm 0.64$ & 0.881 \\
\hline & $\mathrm{DS}+\mathrm{HM}$ & 271 & $2.96 \pm 0.8$ & $3.23 \pm 0.92$ & $-0.1 \pm 0.68$ & 0.017 \\
\hline \multirow{4}{*}{$\mathrm{HbAlC} \%$} & $\mathrm{P}+\mathrm{GM}$ & 271 & $5.68 \pm 0.68$ & $5.59 \pm 0.8$ & $0.24 \pm 0.49$ & $<0.001$ \\
\hline & $\mathrm{P}+\mathrm{HM}$ & 270 & $5.63 \pm 0.66$ & $5.52 \pm 0.77$ & $0.29 \pm 0.7$ & $<0.001$ \\
\hline & $\mathrm{DS}+\mathrm{GM}$ & 278 & $5.64 \pm 0.69$ & $5.49 \pm 0.6$ & $0.32 \pm 0.65$ & $<0.001$ \\
\hline & $\mathrm{DS}+\mathrm{HM}$ & 272 & $5.56 \pm 0.69$ & $5.49 \pm 0.62$ & $0.31 \pm 0.55$ & $<0.001$ \\
\hline \multirow{4}{*}{ LnLEP } & $\mathrm{P}+\mathrm{GM}$ & 268 & $3.15 \pm 0.83$ & $3.13 \pm 0.85$ & $-0.02 \pm 0.47$ & 0.55 \\
\hline & $\mathrm{P}+\mathrm{HM}$ & 269 & $3.13 \pm 0.94$ & $3.16 \pm 0.85$ & $0.03 \pm 0.41$ & 0.298 \\
\hline & $\mathrm{DS}+\mathrm{GM}$ & 275 & $3.16 \pm 0.84$ & $3.12 \pm 0.85$ & $0.08 \pm 0.44$ & 0.003 \\
\hline & $\mathrm{DS}+\mathrm{HM}$ & 271 & $3.16 \pm 0.92$ & $2.99 \pm 0.92$ & $0.16 \pm 0.4$ & $<0.001$ \\
\hline \multirow{4}{*}{ LnINS } & $\mathrm{P}+\mathrm{GM}$ & 271 & $2.32 \pm 0.51$ & $2.48 \pm 0.67$ & $-0.16 \pm 0.66$ & $<0.001$ \\
\hline & $\mathrm{P}+\mathrm{HM}$ & 265 & $2.36 \pm 0.49$ & $2.33 \pm 0.61$ & $0.04 \pm 0.58$ & 0.259 \\
\hline & $\mathrm{DS}+\mathrm{GM}$ & 277 & $2.34 \pm 0.5$ & $2.18 \pm 0.59$ & $0.16 \pm 0.55$ & $<0.001$ \\
\hline & $\mathrm{DS}+\mathrm{HM}$ & 271 & $2.45 \pm 0.52$ & $2.16 \pm 0.63$ & $0.29 \pm 0.62$ & $<0.001$ \\
\hline \multirow{4}{*}{ GLU } & $\mathrm{P}+\mathrm{GM}$ & 270 & $5.03 \pm 1.01$ & $5.27 \pm 1.5$ & $-0.16 \pm 0.94$ & 0.006 \\
\hline & $\mathrm{P}+\mathrm{HM}$ & 268 & $4.95 \pm 1.22$ & $5.05 \pm 1.24$ & $-0.06 \pm 1.05$ & 0.388 \\
\hline & $\mathrm{DS}+\mathrm{GM}$ & 277 & $5.02 \pm 1.25$ & $4.97 \pm 1.12$ & $0.16 \pm 0.82$ & 0.001 \\
\hline & $\mathrm{DS}+\mathrm{HM}$ & 271 & $4.94 \pm 1.07$ & $4.93 \pm 0.99$ & $0.03 \pm 0.84$ & 0.583 \\
\hline \multirow{4}{*}{ HOMAIR } & $\mathrm{P}+\mathrm{GM}$ & 268 & $2.39 \pm 1.73$ & $3.59 \pm 3.75$ & $-0.96 \pm 3.67$ & $<0.001$ \\
\hline & $\mathrm{P}+\mathrm{HM}$ & 265 & $2.3 \pm 1.81$ & $2.86 \pm 2.7$ & $0.64 \pm 2.24$ & 0.435 \\
\hline & $\mathrm{DS}+\mathrm{GM}$ & 275 & $2.48 \pm 2.35$ & $2.38 \pm 2.15$ & $0.32 \pm 2.16$ & 0.013 \\
\hline & $\mathrm{DS}+\mathrm{HM}$ & 270 & $2.36 \pm 2.23$ & $2.35 \pm 2.17$ & $0.64 \pm 2.24$ & $<0.001$ \\
\hline \multirow{4}{*}{ HOMAIS } & $\mathrm{P}+\mathrm{GM}$ & 268 & $0.03 \pm 0.02$ & $0.02 \pm 0.01$ & $-0.0022 \pm 0.01$ & 0.008 \\
\hline & $\mathrm{P}+\mathrm{HM}$ & 269 & $0.03 \pm 0.02$ & $0.02 \pm 0.02$ & $0.002 \pm 0.02$ & 0.037 \\
\hline & $\mathrm{DS}+\mathrm{GM}$ & 275 & $0.03 \pm 0.02$ & $0.03 \pm 0.02$ & $0.0049 \pm 0.02$ & $<0.001$ \\
\hline & $\mathrm{DS}+\mathrm{HM}$ & 271 & $0.03 \pm 0.02$ & $0.03 \pm 0.02$ & $0.0085 \pm 0.02$ & $<0.001$ \\
\hline \multirow{4}{*}{ LnTNF } & $\mathrm{P}+\mathrm{GM}$ & 268 & $3 \pm 0.98$ & $3.15 \pm 0.83$ & $-0.14 \pm 0.73$ & 0.001 \\
\hline & $\mathrm{P}+\mathrm{HM}$ & 269 & $2.99 \pm 1.04$ & $3.01 \pm 0.99$ & $-0.04 \pm 0.8$ & 0.406 \\
\hline & $\mathrm{DS}+\mathrm{GM}$ & 275 & $2.93 \pm 1.04$ & $3.02 \pm 0.98$ & $-0.16 \pm 0.82$ & 0.001 \\
\hline & $\mathrm{DS}+\mathrm{HM}$ & 271 & $2.97 \pm 0.95$ & $3.03 \pm 0.92$ & $-0.12 \pm 0.75$ & 0.006 \\
\hline
\end{tabular}


TABLE 3: Continued.

\begin{tabular}{lcccccc}
\hline & & $N$ & Baseline & 12 months & Annual decline & $0.02 \pm 0.32$ \\
\multirow{3}{*}{ FFA } & P + GM & 271 & $0.47 \pm 0.22$ & $0.5 \pm 0.26$ & 0.215 \\
& P + HM & 269 & $0.48 \pm 0.19$ & $0.51 \pm 0.3$ & 0.901 \\
& DS + GM & 276 & $0.48 \pm 0.21$ & $0.58 \pm 0.27$ & $-0.04 \pm 0.34$ \\
& DS + HM & 269 & $0.45 \pm 0.21$ & $0.56 \pm 0.31$ & $0 \pm 0.36$ & 0.071 \\
\multirow{3}{*}{ LnADI } & P + GM & 268 & $7.71 \pm 0.63$ & $7.66 \pm 0.8$ & $0.01 \pm 0.55$ \\
& P + HM & 269 & $7.73 \pm 0.66$ & $7.75 \pm 0.71$ & $0.03 \pm 0.46$ \\
& DS + GM & 275 & $7.71 \pm 0.67$ & $7.74 \pm 0.69$ & $0.06 \pm 0.46$ & 0.22 \\
& DS + HM & 270 & $7.75 \pm 0.77$ & $7.77 \pm 0.75$ & $0.03 \pm 0.48$ \\
\hline
\end{tabular}

Note. P: placebo; DS: dietary supplements; GM: general management, H: health management.

DS * HM: cross function of dietary supplements and health management.

with/without health management, and the results were shown in Table 4. Effects of with/without dietary supplements intervention on the reduction of CRP, leptin, insulin, TC, LDL, blood sugar, FFA, HOMAIR, and HOMAIS were statistically significant $(p<0.05)$, while the effects of with/without health management on the reduction of leptin, insulin, TG, HOMAIR, and HOMAIS and on the rise of HDL were statistically significant $(p<0.05)$. The interactions between dietary supplements intervention and health management were statistically insignificant $(p>0.05)$ for all the indicators.

3.3. Correlation Analysis. Based on the correlation analysis between weight loss and metabolic changes, the annual declines in C-reactive protein, insulin, total cholesterol (TC), LDL, leptin, triglyceride (TG), blood sugar (GLU), and HOMAIR were positively correlated with weight loss, and the correlation coefficients are statistically significant (Table 5); the annual rises in HOMAIS were positively correlated with weight loss, and the correlation coefficient is statistically significant $(p<0.001)$ (Table 5).

\section{Discussions}

Obesity is a severe problem worldwide currently. Chronic diseases such as hypertension, cardiovascular event, and type II diabetes mellitus are often related to obesity. Weight loss results in drop of blood pressure, improvement of lipid, and decrease of the incidence of diabetes mellitus. Diet, exercise, and behavioral correction are three effective ways that are being used to control overweight and obesity. However, there was no comprehensive study of integrated health management intervention including both dietary supplement and health management on overweight and obesity previously. In this study, the effects of integrated health management intervention including dietary supplements and health management intervention on obese/overweight adults were investigated. The effects were measured by physical indicators changes such as decline of bodyweight, BMI, BFR, waistline, and hipline and the effective weight loss of $5 \%$ in overweight and obese adults. The correlation of dietary supplements and health management intervention on each indicator was studied and interactive effect between them was also studied. Our data showed that integrated health management intervention had the best results on reduction of these key physical indicators. Comparing with other groups, integrated health management intervention showed significant difference, suggesting that combination of dietary supplements and health management intervention was better than dietary supplements or health management intervention alone. These data were consistent with our previous study, in that we also showed the interactive effect between dietary supplements and health management intervention on obese people based on constitutional classification [12].

In addition to observation of the improvement of physical indicators, changes of the metabolic indicators were also studied to find the underlying mechanism of integrated health management intervention on overweight and obesity. Based on the correlation analysis between weight loss and metabolic changes, the annual declines in CRP, insulin, triglyceride, blood sugar, and insulin resistance index were positively correlated with weight loss, and the correlation coefficients were statistically significant. The annual rises in insulin sensitivity index were positively correlated with weight loss, and the correlation coefficient was also statistically significant. These results suggested the possibility of integrated health management intervention, including diet, exercise, and behavioral correction, controlling overweight and obesity by improving different metabolic indicators. In previous studies, weight loss, exercise, and smoking all decreased CRP level [13-15]. Association between aerobic exercise and low levels of high sensitivity-CRP (hs-CRP) was previously observed $[16,17]$. Previous studies also showed that CRP level was related to dietary factors. High intakes of vegetables and fruit were associated with lower levels of circulating hs-CRP [18]. It might be used to explain the lower serum CRP levels in Asian population than the Western populations besides genetic factors [19]. Meta-analysis of randomized controlled trials also suggested dietary factors in regulating serum level of CRP. In obese populations, flaxseed and its derivatives may significantly reduce CRP [16]. Carotenoids and vitamin $\mathrm{C}$ and vitamin $\mathrm{D}$ supplementation significantly decreased the circulating hs-CRP level as shown by meta-analysis [20]. A significant reduction in CRP levels was also seen in vitamin E-treated individuals [21]. In this study, dietary supplements administrated were containing 
TABLE 4: Factorial and interaction analysis of metabolic factors.

\begin{tabular}{|c|c|c|c|}
\hline & Mixed linear model & $F$ & $p$ \\
\hline \multirow{3}{*}{ Lnhs-CRP } & DS & 4.14 & 0.017 \\
\hline & HM & 2.168 & 0.115 \\
\hline & $\mathrm{DS} * \mathrm{HM}$ & 0.31 & 0.734 \\
\hline \multirow{3}{*}{ LnTNF } & DS & 0.764 & 0.466 \\
\hline & HM & 1.733 & 0.177 \\
\hline & $\mathrm{DS} * \mathrm{HM}$ & 0.579 & 0.561 \\
\hline \multirow{3}{*}{ LnADI } & DS & 1.423 & 0.241 \\
\hline & HM & 0.828 & 0.437 \\
\hline & $\mathrm{DS} * \mathrm{HM}$ & 0.638 & 0.505 \\
\hline \multirow{3}{*}{ LnLEP } & DS & 9.676 & 0 \\
\hline & HM & 4.058 & 0.017 \\
\hline & $\mathrm{DS} * \mathrm{HM}$ & 1.191 & 0.304 \\
\hline \multirow{3}{*}{ LnINS } & DS & 35.824 & 0 \\
\hline & $\mathrm{HM}$ & 10.349 & 0 \\
\hline & $\mathrm{DS} * \mathrm{HM}$ & 2.606 & 0.074 \\
\hline \multirow{3}{*}{$\mathrm{TC}$} & DS & 0.363 & 0.696 \\
\hline & $\mathrm{HM}$ & 2.226 & 0.108 \\
\hline & $\mathrm{DS} * \mathrm{HM}$ & 1.048 & 0.351 \\
\hline \multirow{3}{*}{ TG } & DS & 13.974 & 0 \\
\hline & $\mathrm{HM}$ & 4.401 & 0.012 \\
\hline & $\mathrm{DS} * \mathrm{HM}$ & 1.445 & 0.236 \\
\hline \multirow{3}{*}{ HDL-c } & DS & 1.738 & 0.176 \\
\hline & $\mathrm{HM}$ & 6.567 & 0.001 \\
\hline & $\mathrm{DS} * \mathrm{HM}$ & 2.609 & 0.074 \\
\hline \multirow{3}{*}{ LDL-c } & DS & 3.31 & 0.037 \\
\hline & $\mathrm{HM}$ & 0.459 & 0.632 \\
\hline & $\mathrm{DS} * \mathrm{HM}$ & 2.395 & 0.092 \\
\hline \multirow{3}{*}{ GLU } & DS & 9.095 & 0 \\
\hline & $\mathrm{HM}$ & 0.738 & 0.478 \\
\hline & $\mathrm{DS} * \mathrm{HM}$ & 2.455 & 0.086 \\
\hline \multirow{3}{*}{ FFA } & DS & 5.746 & 0.003 \\
\hline & HM & 0.833 & 0.435 \\
\hline & $\mathrm{DS} * \mathrm{HM}$ & 1.115 & 0.328 \\
\hline \multirow{3}{*}{$\mathrm{HbAlC} \%$} & DS & 1.349 & 0.26 \\
\hline & $\mathrm{HM}$ & 1.319 & 0.268 \\
\hline & $\mathrm{DS} * \mathrm{HM}$ & 1.21 & 0.298 \\
\hline \multirow{3}{*}{ HOMAIR } & DS & 25.728 & 0 \\
\hline & HM & 6.568 & 0.001 \\
\hline & $\mathrm{DS} * \mathrm{HM}$ & 2.836 & 0.059 \\
\hline \multirow{3}{*}{ HOMAIS } & DS & 28.597 & 0 \\
\hline & HM & 7.57 & 0.001 \\
\hline & $\mathrm{DS} * \mathrm{HM}$ & 0.064 & 0.938 \\
\hline
\end{tabular}

Note. Single-factor variance analysis was used, where $p<0.05$ denotes a statistically significant difference.

P: placebo; DS: dietary supplements; GM: general management, $H$ : health management.

DS * HM: cross function of dietary supplements and health management.

fibers and other special supplements that could regulate constitution according to Chinese Medicine. They might control obesity by improving metabolic indicators. In fact, CRP level significantly decreased after different interventions
TABLE 5: Correlation between weight loss and metabolic indicators and correlation factor.

\begin{tabular}{lcc}
\hline & \multicolumn{2}{c}{ Over all } \\
\hline Decline in hs-CRP & 0.234 & $p$ \\
Decline in TC & 0.11 & $<0.001$ \\
Decline in TG & 0.285 & $<0.001$ \\
Rise in HDL & 0.082 & $<0.001$ \\
Decline in LDL & 0.093 & 0.007 \\
Decline in GLU & 0.134 & 0.002 \\
Decline in HbA1C & 0.1 & $<0.001$ \\
Decline in LnINS & 0.343 & 0.001 \\
Decline in LnLEP & 0.096 & $<0.001$ \\
Rise in HOMAIR & 0.258 & 0.002 \\
Rise in HOMAIS & 0.295 & 0.002 \\
Rise in LnADI & 0.02 & $<0.001$ \\
Decline in FFA & -0.012 & 0.514 \\
Decline in LnTNF & 0.027 & 0.687 \\
\hline
\end{tabular}

Note. Multiple logistic regression analysis was used for testing the correlation between weight loss and metabolic indicators and correlation factor.

in all four groups, suggesting both dietary supplements and health managements had effect on CRP decline. In addition, the annual decline in CRP was best in the dietary supplements + health management group, suggesting that intergraded health management had better effect on the annual decline of CRP. However, data analysis showed that dietary supplements intervention on the reduction of CRP was statistically significant while health management on the reduction of CRP was statistically insignificant and the interaction between dietary supplements intervention and health management was statistically insignificant. These results suggested that CRP decline in this one-year study was more related to the dietary factors other than health management. The same results were seen in blood glucose, LDL, and FFA. From the analysis of other metabolic indicators such as leptin, insulin, HOMAIR, HOMAIS, and TG, both dietary supplements intervention and health management intervention on the reduction of these indicators were statistically significant. These results supported that integrated health management could control overweight and obesity by improving different metabolic indicators through either dietary supplements intervention or health management intervention or both.

Moreover, all the metabolic indicators including TC, TG, FFA, GLU, insulin, HOMAIR, HOMAIS, leptin, HbAlC, HDL, LDL, and TNF were previously proved to have the relationship with chronic disease of hypertension, diabetes mellitus, and cardiovascular disease [22, 23]. CRP has also been intensively studied and meta-analysis for the relationship of CRP with hypertension, cardiovascular disease, and diabetes mellitus suggested its role in mediating these chronic diseases $[24,25]$. Therefore, the parallel changes of these metabolic indicators might suggest a crosstalk between them. CRP could be the intermediate factor between these indicators and chronic diseases such as hypertension, diabetes mellitus, and cardiovascular diseases as well as obesity. 
Evidences supporting this hypothesis could also be found in previous studies. In a study on the relationship between obesity and CRP in the children, the results showed that the obese children had significantly increased CRP. In addition, leptin and insulin also significantly increased comparing to the control group. In this study, BMI had positive correlation with CRP and negative correlation with insulin sensitive index. Leptin had positive correlation between insulin and BMI while it had negative correlation with insulin sensitive index. These suggested that obese children may have insulin resistance and leptin resistance. Both CRP and leptin could play important role in the process of obesity. In overweight and obese adults, elevated level of CRP was also seen [26, 27]. In this study, higher BMI was associated with higher CRP concentrations, suggesting a state of low-grade systemic inflammation in overweight and obese adults. In the obese persons, expanded adipose tissue secreted proinflammatory cytokines which enhance hepatic synthesis of CRP. CRP could bind to LDL, VLDL, and oxidized LDL and promote complement activation. The interaction of CRP and LDL might further affect lipid metabolism and promote obese and other chronic diseases. Studies also supported that CRP had direct proinflammatory effect and was an independent risk factor in a variety of cardiovascular diseases and might be used as a biomarker to predicate the risk of cardiovascular disease and to predicate the long-term outcome of acute coronary syndrome [28-30]. Therefore, CRP could function as an inflammatory marker and mediated the function of lipid protein in obese and cardiovascular disease. Correlation studies on obesity and type II diabetes also showed that significantly high level of CRP was seen in obese people both in diabetes group and control group, suggesting that CRP might be the major link between obesity and type II diabetes. Therefore, integrated health management intervention including both dietary supplements and health managements might affect metabolic indicators directly or indirectly by CRP to control obesity. CRP level might be used as a promising marker to assess improvements in obesity.

\section{Competing Interests}

The authors declare there is no conflict of interests.

\section{Acknowledgments}

The authors would like to thank Dr. David Eisenberg of Harvard T.H. Chan School of Public Health for his generous help in designing of this research project and data statistics.

\section{References}

[1] J. Lu, Y. Bi, and G. Ning, "Curbing the obesity epidemic in China," The Lancet Diabetes \& Endocrinology, vol. 4, no. 6, pp. 470-471, 2016.

[2] H. Xue, Y. Wu, X. Wang, and Y. Wang, "Time trends in fast food consumption and its association with obesity among children in China," PLoS ONE, vol. 11, no. 3, Article ID e0151141, 2016.

[3] D. C. Coitinho, J. A. Rivera, R. Uauy, Z.-Y. Ding, M. T. Ruel, and P.-G. Svensson, "Emerging nutrition challenges: policies to tackle under-nutrition, obesity and chronic diseases," World Hospitals and Health Services, vol. 44, no. 3, pp. 45-48, 2008.

[4] J. Song, S. Chen, X. Liu, H. Duan, J. Kong, and Z. Li, "Relationship between C-reactive protein level and diabetic retinopathy: a systematic review and meta-analysis," PLoS ONE, vol. 10, no. 12, Article ID e0144406, 2015.

[5] M. J. Hosseinzadeh-Attar, A. Golpaie, M. Foroughi, F. Hosseinpanah, S. Zahediasl, and F. Azizi, "The relationship between visfatin and serum concentrations of $\mathrm{C}$-reactive protein, interleukin 6 in patients with metabolic syndrome," Journal of Endocrinological Investigation, vol. 39, no. 8, pp. 917-922, 2016.

[6] K. M. Beavers, D. P. Beavers, J. J. Newman et al., "Effects of total and regional fat loss on plasma CRP and IL- 6 in overweight and obese, older adults with knee osteoarthritis," Osteoarthritis and Cartilage, vol. 23, no. 2, pp. 249-256, 2015.

[7] M. Visser, L. M. Bouter, G. M. McQuillan, M. H. Wener, and T. B. Harris, "Elevated C-reactive protein levels in overweight and obese adults," The Journal of the American Medical Association, vol. 282, no. 22, pp. 2131-2135, 1999.

[8] W. M. Brown, G. W. Davison, C. M. McClean, and M. H. Murphy, "A systematic review of the acute effects of exercise on immune and inflammatory indices in untrained adults," Sports Medicine-Open, vol. 1, no. 1, article 35, 2015.

[9] S. Saboori, S. Shab-Bidar, J. R. Speakman, E. Yousefi Rad, and K. Djafarian, "Effect of Vitamin E supplementation on serum Creactive protein level: a meta-analysis of randomized controlled trials," European Journal of Clinical Nutrition, vol. 69, no. 8, pp. 867-873, 2015.

[10] N. Bijeh, S. A. Hosseini, and K. Hejazi, "The effect of aerobic exercise on serum C-reactive protein and leptin levels in untrained middle-aged women," Iranian Journal of Public Health, vol. 41, pp. 36-41, 2012.

[11] M. Henderson, A. Benedetti, and K. Gray-Donald, "Dietary composition and its associations with insulin sensitivity and insulin secretion in youth," British Journal of Nutrition, vol. 111, no. 3, pp. 527-534, 2014.

[12] W. Q. Zhu Yanbo, W. Chengyu, P. Guoming et al., "A Logistic regression analysis: relationship between Traditional Chinese Medicine body constitution type classification and oveweight and obesity in 18805 Chinese adults," Journal of Chinese Integrative Medicine, vol. 8, no. 11, pp. 1023-1028, 2010.

[13] J. Genest, "C-reactive protein: risk factor, biomarker and/or therapeutic target?" Canadian Journal of Cardiology, vol. 26, pp. 41A-44A, 2010.

[14] A. Nanri, M. A. Moore, and S. Kono, "Impact of C-reactive protein on disease risk and its relation to dietary factors: literature review," Asian Pacific Journal of Cancer Prevention, vol. 8, no. 2, pp. 167-177, 2007.

[15] H. S. Park, J. Y. Park, and R. Yu, "Relationship of obesity and visceral adiposity with serum concentrations of CRP, TNF- $\alpha$ and IL-6," Diabetes Research and Clinical Practice, vol. 69, no. 1, pp. 29-35, 2005.

[16] G. Y. Ren, C. Y. Chen, G. C. Chen et al., "Effect of flaxseed intervention on inflammatory marker C-reactive protein: a systematic review and meta-analysis of randomized controlled trials," Nutrients, vol. 8, no. 3, p. 136, 2016.

[17] E. Selvin, N. P. Paynter, and T. P. Erlinger, "The effect of weight loss on C-reactive protein: a systematic review," Archives of Internal Medicine, vol. 167, no. 1, pp. 31-39, 2007.

[18] Y.-W. Xiao, X.-M. Huang, Y.-X. Deng et al., "Correlation of obesity with C-reactive protein, leptin and insulin sensitive 
index in children," Zhongguo Dang Dai Er Ke Za Zhi, vol. 8, no. 1, pp. 24-26, 2006.

[19] N. Rifai and P. M. Ridker, "Population distributions of Creactive protein in apparently healthy men and women in the United States: implication for clinical interpretation," Clinical Chemistry, vol. 49, no. 4, pp. 666-669, 2003.

[20] J.-Y. Dong, P. Wang, K. He, and L.-Q. Qin, "Effect of soy isoflavones on circulating C-reactive protein in postmenopausal women: meta-analysis of randomized controlled trials," Menopause, vol. 18, no. 11, pp. 1256-1262, 2011.

[21] L.-M. Zhou, J.-Y. Xu, C.-P. Rao, S. Han, Z. Wan, and L.Q. Qin, "Effect of whey supplementation on circulating Creactive protein: a meta-analysis of randomized controlled trials," Nutrients, vol. 7, no. 2, pp. 1131-1143, 2015.

[22] F. Leyva, I. F. Godsland, M. Worthington, C. Walton, and J. C. Stevenson, "Factors of the metabolic syndrome: baseline interrelationships in the first follow-up cohort of the HDDRISC study (HDDRISC-1)," Arteriosclerosis, Thrombosis, and Vascular Biology, vol. 18, no. 2, pp. 208-214, 1998.

[23] S. Barquera, I. Campos-Nonato, C. Carrión-Rábago et al., "Methodology for the analysis of type 2 diabetes, metabolic syndrome and cardiovascular disease risk indicators in the ENSANUT 2006," Salud Pública de México, vol. 52, supplement 1, pp. S4-S10, 2010.

[24] S. Yamada, T. Gotoh, Y. Nakashima et al., "Distribution of serum C-reactive protein and its association with atherosclerotic risk factors in a Japanese population: jichi medical school cohort study," American Journal of Epidemiology, vol. 153, no. 12, pp. 1183-1190, 2001.

[25] M. G. Kim, B. H. Cho, S. W. Chae, T. S. Park, and D. S. Kim, "What do very low plasma concentrations of high-sensitivity C-reactive protein (hs-CRP) mean among healthy middle-aged Koreans?” Journal of Lifestyle Medicine, vol. 5, pp. 14-20, 2015.

[26] F. B. Hu, "The Mediterranean diet and mortality-olive oil and beyond," The New England Journal of Medicine, vol. 348, no. 26, pp. 2595-2596, 2003.

[27] A. Trichopoulou, T. Costacou, C. Bamia, and D. Trichopoulos, "Adherence to a Mediterranean diet and survival in a Greek population," The New England Journal of Medicine, vol. 348, no. 26, pp. 2599-2608, 2003.

[28] O. Yousuf, B. D. Mohanty, S. S. Martin et al., "High-sensitivity Creactive protein and cardiovascular disease: a resolute belief or an elusive link?" Journal of the American College of Cardiology, vol. 62, no. 5, pp. 397-408, 2013.

[29] D. Y. Kamath, D. Xavier, A. Sigamani, and P. Pais, "High sensitivity C-reactive protein (hsCRP) \& cardiovascular disease: an Indian perspective," Indian Journal of Medical Research, vol. 142, pp. 261-268, 2015.

[30] J. Choi, L. Joseph, and L. Pilote, "Obesity and C-reactive protein in various populations: a systematic review and meta-analysis," Obesity Reviews, vol. 14, no. 3, pp. 232-244, 2013. 


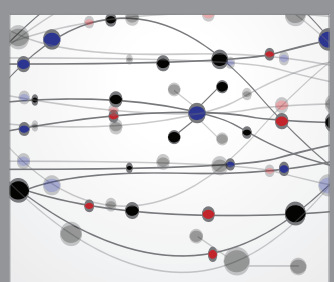

The Scientific World Journal
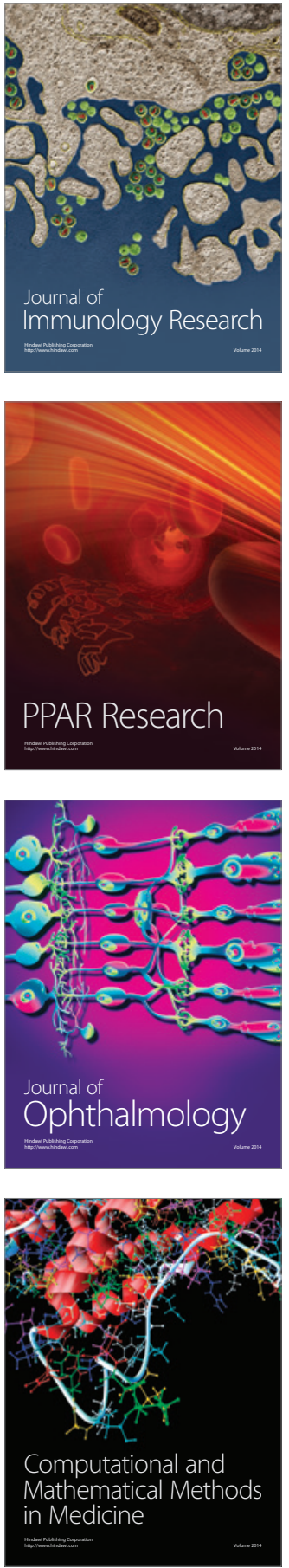

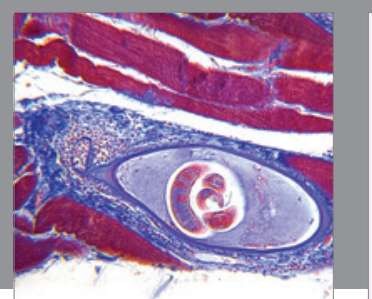

Gastroenterology Research and Practice
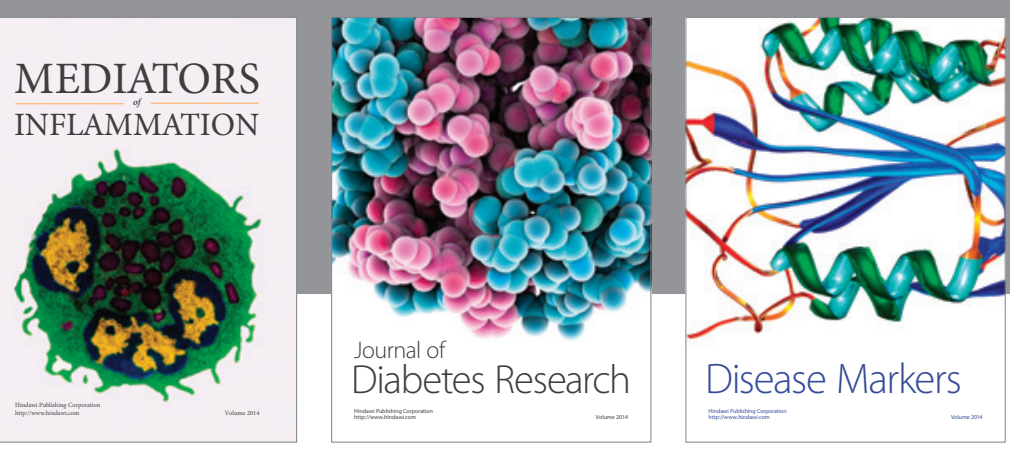

Disease Markers

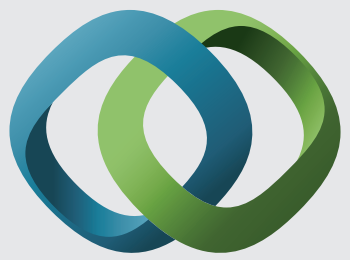

\section{Hindawi}

Submit your manuscripts at

https://www.hindawi.com
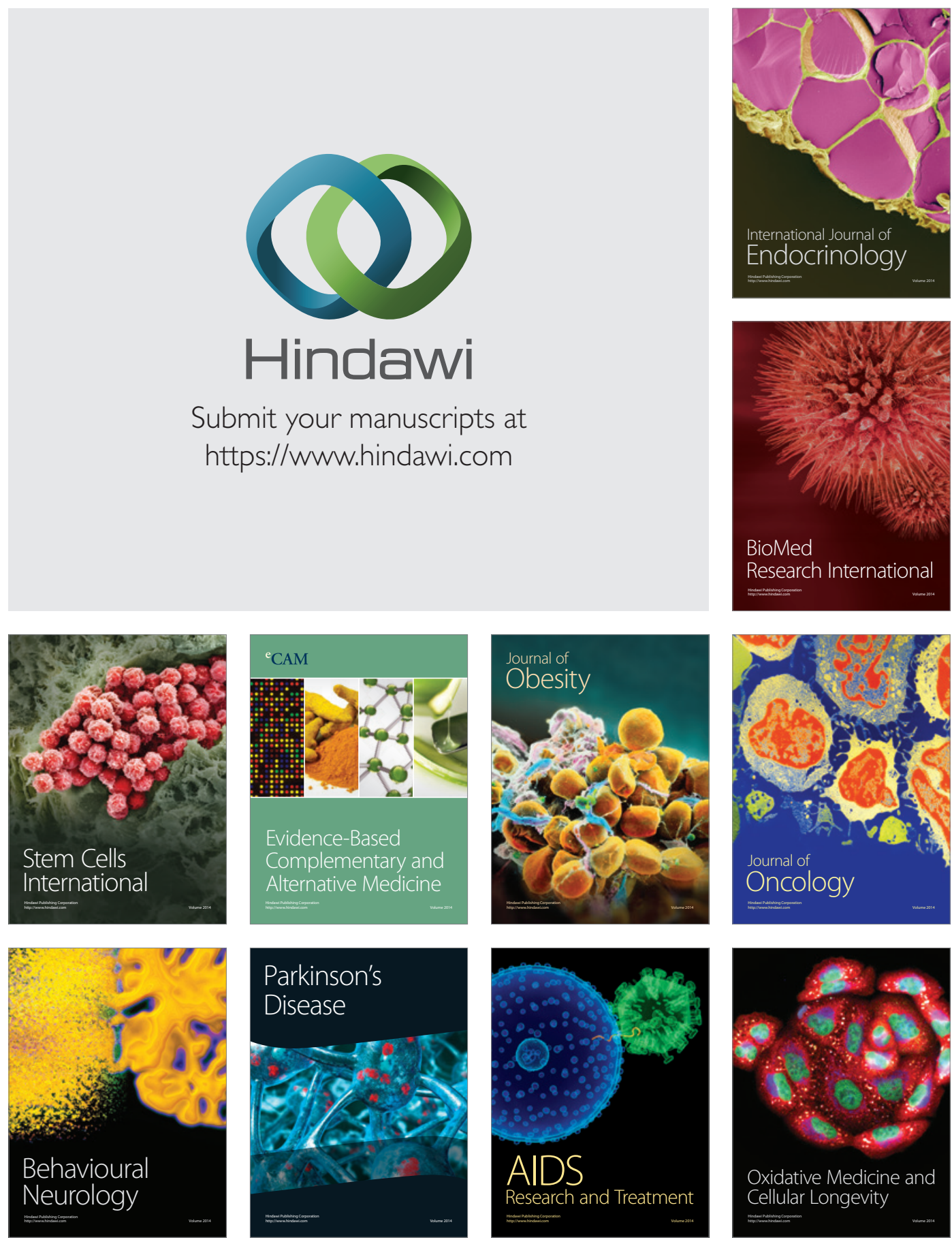